\title{
Efficacy of Single Session Sclerotherapy with Undiluted Polydocanol, in Complete Elimination of Cutaneous as Well as Mucosal Venous Malformations of the Maxillofacial Region
}

\section{Priya Jeyaraj*}

Classified Specialist (Oral and Maxillofacial Surgery), Deputy Commandant; Officer in Charge Trauma and Rehabilitation, Command Military Dental Centre (Northern Command), Jammu and Kashmir, India

\begin{abstract}
Haemangiomas and Vascular malformations of the maxillofacial region are alarming lesions which cause considerable cosmetic deformity and psychological problems. Their surgical excision is difficult and fraught with complications like intraoperative blood loss, incomplete excision, disfigurement and recurrence. Sclerotherapy is the percutaneous or permucosal intralesional endovascular instillation of an irritant agent/sclerosant, which helps to reduce their size and make them more amenable to surgical excision later.
\end{abstract}

This case series demonstrates the efficacy and safety of a single session Sclerotherapy with undiluted Polydocanol in the management of medium sized vascular malformations involving the maxillofacial regions (lip, nose and buccal mucosa), which completely resolved within four weeks of the intralesional injection, without any need for surgical excision. The gratifying results achieved, support the fact that single session sclerotherpy with Polydocanol may be considered as the primary modality in the treatment of these challenging lesions.

Keywords: Vascular anomalies; Vascular malformation (VM); Haemangioma; Sclerotherapy; Polydocanol

\section{Introduction}

Vascular lesions or anomalies fall under two major categories: vascular tumors, also referred to as haemangiomas, and vascular malformations (VMs) [1]. Haemangiomas are true vascular neoplasms, usually seen in infancy and childhood, containing an increased number of normal or abnormal blood vessels lined with thickened subendothelial basement membrane laminas, and hypercellular endothelial cells. They are characterized clinically by a rapid neonatal growth until the age of 6-8 months (proliferative phase) and then a slow regression by the age of 5-10 years (involuting phase), characterized by diminishing cellularity, interstitial fibrosis and fibrofatty replacement.

Vascular malformations, on the other hand, are developmental anomalies, containing abnormally formed channels that are lined by quiescent endothelium, which may or may not be obvious at birth. They grow commensurate with the growth of the child, persisting into adulthood, and never regress or involute. They are quite common among the adult population and can affect up to $5 \%$ of them [2]. VMs infiltrate skin, muscles, joints, organ systems and sometimes even bones. Symptoms are dependent on the anatomic location of the lesion and can have a great impact on the patient's quality of life. Pain and swelling are common symptoms associated with all VMs. With craniofacial lesions, cosmetic disfigurement may be more debilitating than functional limitations $[3,4]$ often leading to psychological discomfort and feelings of embarrassment, anxiety, low self-esteem, and antisocial behavior. Moreover, these lesions are often associated with recurrent bleeding episodes, secondary infections and ulcerations. From these perspectives, the treatment of VMs is more than just a cosmetic concern, but also has a high degree of medical relevance.

VMs were considered to be best managed by surgical excision. However, surgery is often cumbersome, time consuming and associated with technical difficulties and complications, such as inadvertent injury to important head and neck structures, intraoperative bleeding and exsanguination. It may also leave behind scar marks, or residual lesions. Furthermore, cosmetic disfigurement or recurrence may result from incomplete or inappropriate excision

Over the years, treatment of vascular lesions has substantially changed, and less invasive therapies with improved results have evolved. These advancements allow for safer, more effective treatments and increase the level of comfort for patients and physicians. Cryosurgery, compression and radiation came into vogue, and have now been replaced by endovenous laser therapy $[5,6]$ flash lamp pulsed laser, intralesional injection of fibrosing agents, interferon alpha-2b [7], electrocoagulation and radiofrequency ablation. Endovascular techniques like percutaneous/permucosal Sclerotherapy and Microembolization [8] are increasingly being used in the treatment of these difficult lesions making them more amenable to easier surgical excision.

For superficial lesions, lasers have become first-line therapy, as they specifically target the natural absorption peaks of oxyhemoglobin and deoxyhemoglobin to create thermal energy. The selective endogenous destruction is associated with only minimal damage to surrounding tissues and allows for the treatment of superficial vascular lesions without scarring. Long pulsed dye lasers (PDLs) for the treatment of vascular lesions is another important development [9]. An interesting discovery in the treatment of vascular lesions is the use of

${ }^{*}$ Corresponding author: Jeyaraj $\mathrm{P}$, Classified Specialist (Oral and Maxillofacia Surgery), Deputy Commandant; Officer in Charge Trauma and Rehabilitation, Command Military Dental Centre (Northern Command), Jammu and Kashmir India, Tel: +91-9596840303; E-mail: jeyarajpriya@yahoo.com

Received June 09, 2017; Accepted July 08, 2017; Published July 16, 2017

Citation: Jeyaraj P (2017) Efficacy of Single Session Sclerotherapy with Undiluted Polydocanol, in Complete Elimination of Cutaneous as Well as Mucosal Venous Malformations of the Maxillofacial Region. J Cytol Histol 8: 464. doi: 10.4172/21577099.1000464

Copyright: () 2017 Jeyaraj P. This is an open-access article distributed under the terms of the Creative Commons Attribution License, which permits unrestricted use, distribution, and reproduction in any medium, provided the original author and source are credited. 
Citation: Jeyaraj P (2017) Efficacy of Single Session Sclerotherapy with Undiluted Polydocanol, in Complete Elimination of Cutaneous as Well as Mucosal Venous Malformations of the Maxillofacial Region. J Cytol Histol 8: 464. doi: 10.4172/2157-7099.1000464

the beta-blocker propranolol, especially to treat problematic infantile haemangiomas $[10,11]$.

Sclerotherapy is a popular, minimally invasive, simple and cost effective procedure, widely used in the management of hemorrhoids, superficial lower extremity telangiectasias and varicose, reticulate veins other venous abnormalities [12]. It has also been found to be effective in Craniofacial haemangiomas and VMs, and can be performed in the outpatient clinic [13] For smaller lesions, sclerotherapy alone can lead to cure or significant reduction in size while for larger lesions, sclerotherapy may facilitate surgery by decreasing lesion size and blood loss [14-16]. It involves direct injection of a small amount of an irritant solution/sclerosant (such as sodium tetra decyl sulphate, $5 \%$ phenol, sodium morrhuate, sodium psylliate, sodium citrate, invert sugar, absolute alcohol, boiling water, hypertonic saline or hypertonic dextrose) into the abnormally dilated vessels and application of compression to maintain the contact of the intravascular solution with the endothelial cells.

The mechanism of action of a Sclerosant includes a localized inflammatory reaction with swelling, disruption and destruction of the endothelium of the vessel wall, causing platelets and cellular debris to attach to the lining of the vessels, followed by formation of an intravascular coagulum leading to obliterative thrombosis causing the blood vessel to clot and subsequent fibrosis of the Vascular/endothelial spaces, eventually leading to the disappearance of the treated vessels by histiocytic digestion, with consequent regression of the lesion [17]. Upon injection of a sclerosant, there are three zones of action. In Zone 1 , vascular endothelium is irreversibly injured: The vessel will be fully sclerosed and eventually will be completely replaced by fibrous tissue. In Zone 2, vascular endothelium is injured, and the vessel will be partially or completely thrombosed but will eventually recanalize. In Zone 3 , the sclerosant will be diluted below its injurious concentration, and there will be no endothelial injury [18]. Advantages of a Sclerotherapy agent are that it is simple and inexpensive, entailing no loss of blood, and be carried out on an outpatient basis with no need for hospitalization. Disadvantages are occasional anaphylactic reactions, post-operative pain and burning sensation, local swelling, inflammation and tissue necrosis, peripheral nerve injury, hemoglobinuria, thromboembolism, infection and delayed muscle fibrosis [19]. The application should always be carried out in the center and deeper portion of the tumor in order to avoid extensive tissue necrosis of adjacent normal areas [20]. This care was taken during the application of the sclerosing agent in the present case series.

\section{Case Series}

\section{Case 1}

A 32 year old female patient reported with the complaints of a reddish-purple, bulging discoloration of the inside of her cheek and gums on the right side. History revealed that the discoloration had been present since childhood, although it could not be ascertained if it was present at birth or during infancy. It had persisted throughout childhood, adolescence and adulthood, without any rapid increase or decrease in its size. It was only recently that she had noticed it increasing in size, particularly after delivery of a child eight months back. She also gave a history of being on oral contraceptive pills for the last four months. She also complained of occasional pain and recurrent episodes of bleeding from the region, especially when it was accidentally traumatized during brushing or eating or when she inadvertently bit it.

On examination, a well demarcated, deep bluish - purple, raised, irregularly shaped patch was observed on the right buccal mucosa (Figure 1A and 1B), measuring approximately $6 \mathrm{~cm} \times 4 \mathrm{~cm}$, extending anteriorly upto the angle of the mouth and posteriorly upto the second molar region. Superiorly and inferiorly, the patch was continuous with the buccal vestibule and its reflection into the alveolar and gingival mucosa in the right upper posterior quadrant (Figure 1A and $1 \mathrm{~B}$ ).
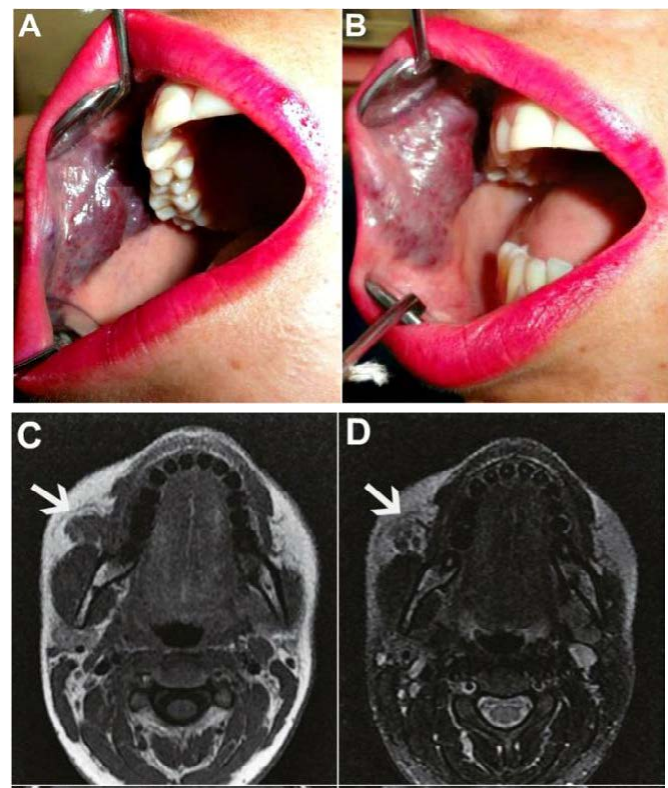

D
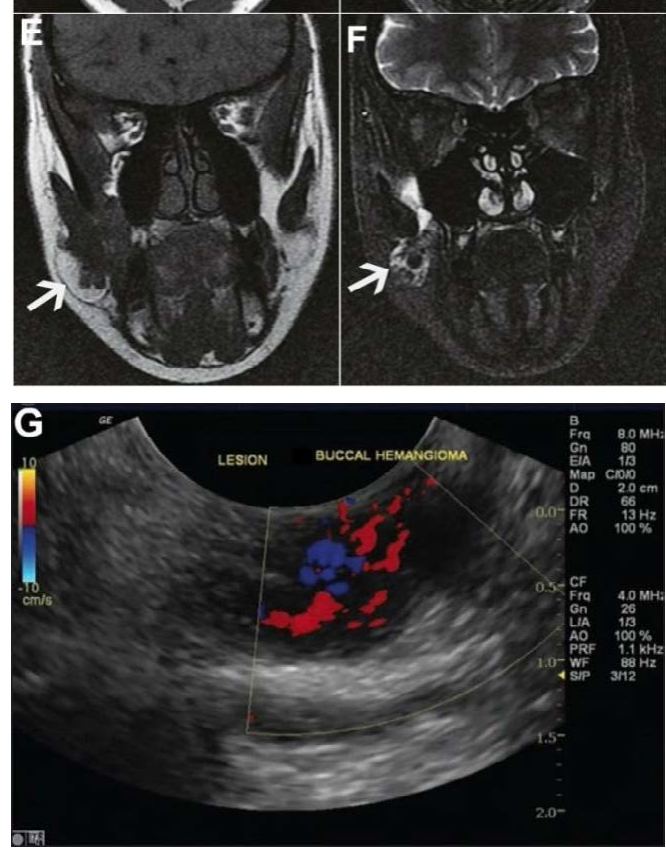

Figure 1: (A-G) A well demarcated, deep bluish - purple, raised, irregularly shaped, friable and pebbly surfaced patch on the right buccal mucosa, which was continuous with the buccal vestibule and its reflection into the alveolar and gingival mucosa in the right upper posterior quadrant. (C and D) Magnetic Resonance Imaging MRI Axial sections (T1 and T2 weighted images with Fat Suppression and Fat saturation/enhancement, respectively) showing an enhancing lesion in the Right Buccal sulcus, as depicted by the white arrows. (E and F) MRI Coronal sections (T1 and T2 weighted images with Fat Suppression and Fat enhancement, respectively) showing rapid and marked enhancement of a mass in the right buccal space and vestibule, as depicted by the white arrows, indicative of a the VM. (G) Ultrasound color Doppler study showing the hypo-echoic region with multiple tiny cystic areas and internal vascularity within the lesion of the buccal mucosa. 
Citation: Jeyaraj P (2017) Efficacy of Single Session Sclerotherapy with Undiluted Polydocanol, in Complete Elimination of Cutaneous as Well as Mucosal Venous Malformations of the Maxillofacial Region. J Cytol Histol 8: 464. doi: 10.4172/2157-7099.1000464

The margins of the lesion were well demarcated and distinct, being at a slightly elevated level from the adjacent unaffected buccal mucosa. The surface appeared bluish purple with elevated deep red speckled spots scattered throughout, giving the lesion a pebbled appearance. There was no evidence of inflammation, infection, ulceration or any other secondary change. On palpation, the surface of the discolored patch was mildly raised, soft and exhibited a pebbly texture. There was no visible or palpable pulsation or bruit over the lesion. The area was extremely friable and bled readily upon probing, particularly from the gingival sulci.

FNAC could not be carried out as even mild probing gave rise to an immediate and ready ooze of blood. PT, pTTK and INR levels were all within normal limits as was the remaining blood and urine profile. The patient was not hypertensive and his blood sugar was within normal limits. MRI (T1 and T2 weighted images with Fat Suppression and Fat enhancement, respectively (Figure 1E and $1 \mathrm{~F}$ ) showed a space occupying lesion involving the buccal vestibule on the right. Ultrasound color Doppler study (Figure 1F) demonstrated a hypo-echoic region with multiple tiny cystic areas and internal vascularity within the lesion, indicative of a slow flow lesion consisting of anomalous channels suggestive of a vascular malformation or a haemangioma.

Differential diagnosis included a hemangioma or a vascular malformation. As the lesion was present since childhood (most likely since birth) and had persisted throughout adolescence and adulthood, growing steadily, without either a rapidly proliferative phase or an involution phase, it was more likely to be a vascular malformation than a residual haemangioma. Moreover, a distinct history of an increase in size of the lesion post-delivery, pointed to the possibility of hormonal changes in the patient to be a possible triggering factor. The patient also gave a history of being on oral contraceptive medication for the past four months, during which she had noticed increase in the lesion's size as well as bleeding from it. Oral contraceptives are an established triggering factor of vascular malformations. As there was no visible pulsation or palpable bruit or warmth over the lesion, it was most likely a low flow vascular malformation. Capillary malformations usually present as telangiectasias or the "Port wine stain" while lymphatic malformations are usually colourless. Hence the bluish-purple mass, which was soft, engorged and compressible, was most likely a venous malformation. Thus, history and clinical presentation led to a provisional diagnosis of a venous malformation.

The treatment plan was to first employ Sclerotherapy with Polydocanol to reduce the size and extent of the lesion, followed by its excision in toto, both procedures to be carried out in an outpatient setting.

The lesion was first isolated using black braided silk sutures to prevent sclerosant spill and to reduce its diffusion into the adjacent unaffected mucosa (Figure 1C and 2D). Immediately the lesion was found to become engorged and enlarged in size (Figure 1C and 2D). The pebbly texture became more pronounced. Dilated veins could be seen protruding just beneath the overlying mucosa of the lesion. $3 \mathrm{cc}$ of undiluted Polydocanol $(3 \mathrm{mg}$ ) was slowly injected into the sides as well as the center of the lesion, using $1 \mathrm{cc}$ syringes after first infiltrating the area with a local anesthetic. Immediately following the injection, the lesion swelled and became tense (Figure $1 \mathrm{E}$ and $1 \mathrm{~F}$ ). There was no uncontrolled bleeding from the permucosal puncture sites, other than mild ooze which soon stopped. The silk sutures were left in situ. The patient was put on anti-inflammatory and analgesic medications for five days and cold compress was advised externally over the cheek region.
On the fifth day following sclerosant injection, there was observed considerable local edema and swelling of the cheek, in the region of the lesion (Figure 2C and 2D). Intraorally, the area of the vascular lesion appeared grayish black in colour, with intraoral necrosis and sloughing of mucosal tissue from it (Figure 2A and 2B). Pain was not severe and was effectively managed with analgesics. The patient was also started in a course of antibiotics. The sutures were removed on the fifth day. In the second week, the swelling, inflammation and sloughing of tissues began to subside. By the third week the swelling had completely regressed and the area of whitish slough was restricted to the center of the area (Figure $2 \mathrm{E}-2 \mathrm{H}$ ).

There was a complete and total resolution of the vascular lesion by the fourth week following the single session Sclerotherapy, with practically no evidence of it in the healed buccal as well as alveolar and gingival mucosae (Figure 3I-3L). So much so, that there was absolutely no need to take the patient up for excision of the residual lesion (Figure 3).

There were no complications such as infection at the injection site or necrosis of the skin in that region. Colour Doppler Ultrasound of the right cheek was performed one and two months after sclerotherapy, which showed a progressive reduction in size of the hypo-echoic region as well as diminished vascularity within it, indicative of successful resolution of the VM (Figure 4A and 4B). The patient was followed up for a period of two years and there was no recurrence of the vascular lesion.
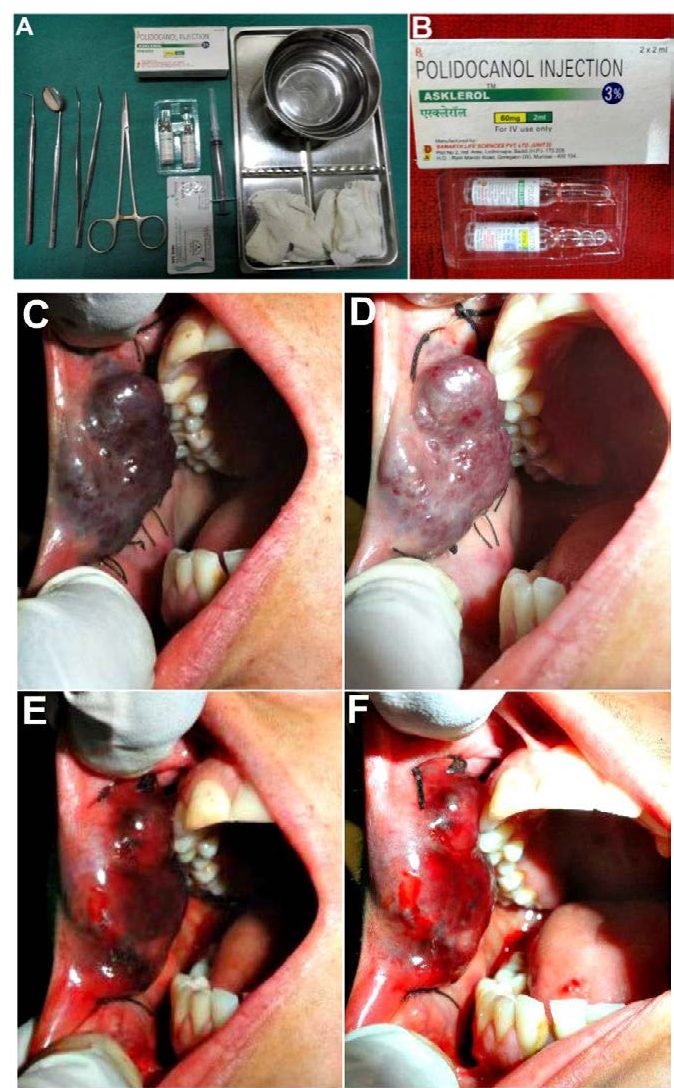

Figure 2: (A-F) Armamentarium required for Intralesional injection of Polydocanol ( $C$ and $D$ ) Lesion observed to become engorged and enlarged in size immediately following its isolation using black braided silk sutures, performed to prevent sclerosant spill into the adjacent unaffected mucosa. ( $E$ and F) $2 \mathrm{cc}$ of undiluted Polydocanol $(60 \mathrm{mg})$ was slowly injected into the sides as well as the center of the lesion, causing the lesion to become tense with blood oozing from the sites of needle pricks. 

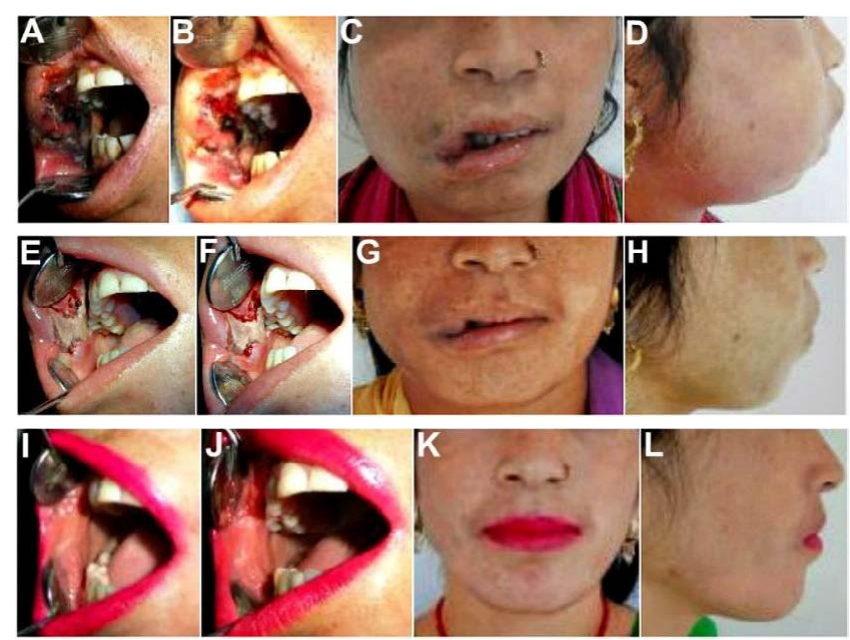

Figure 3: (A-D) Intraoral necrosis and sloughing of mucosal tissue at the site of sclerosant injection, and considerable local edema and swelling of the cheek seen after 5 days of the single session sclerotherpy. (E-H) By the third week the area of whitish grey slough was restricted to the center of the area and the swelling of the cheek and lips had regressed. (I-L) Complete elimination and total resolution of the vascular lesion by the fourth week following the single session Sclerotherapy, with practically no evidence of it in the healed bucca as well as alveolar and gingival mucosae. No residual scarring or discoloration was evident.
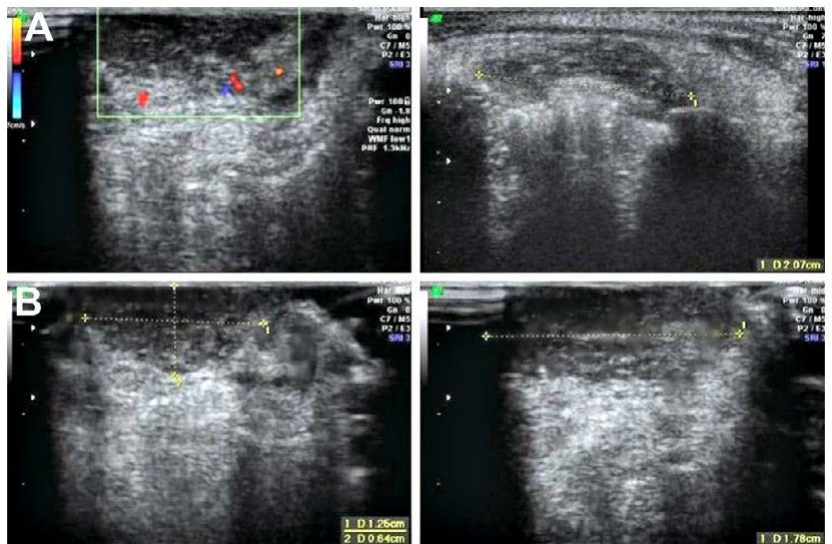

Figure 4: Colour Doppler Ultrasound of the right cheek performed (A) One month after Sclerotherapy; and (B) Two months after sclerotherapy, showing reduction in size of the hypo-echoic region as well as diminished vascularity within it, indicative of successful resolution of the VM.

\section{Case 2}

A 45 year old male patient reported with a longstanding, painless, well demarcated raised, deep purplish lesion on the right side of his nose (Figure 5A-5C). It was approximately $4 \mathrm{~cm} \times 3 \mathrm{~cm}$ in size, had a pebbly texture and blanched on pressure. History revealed that it had been present since childhood, slowly increasing in size over the years. It bled profusely on occasions especially on being rubbed inadvertently with a towel after washing the face. Blood and urine profiles were all within normal limits, and the patient had no other co-morbidities. Non Contrast Computed Tomography of the maxillofacial region (Figure 5D) revealed a soft tissue swelling adjacent to the nose on the right side. Color Doppler Ultrasound image of the region (Figure 5E) demonstrated a mildly increased density of blood vessels within the lesion suggestive of a slow flow VM. Contrast enhanced Magnetic Resonance Angiography was carried out (Figure 5F), which showed a vascular lesion involving branches of the Facial artery supplying lateral aspect of the nose.

$2 \mathrm{cc}$ of undiluted Polydocanol $(2 \mathrm{mg})$ was slowly injected percutaneously into the sides as well as the center of the lesion, using $1 \mathrm{cc}$ syringes after first infiltrating the area with a local anesthetic. There was a mild ooze of blood from the cutaneous puncture sites, which stopped after application of local pressure over the area for 3 minutes. Local edema and swelling developed over the next five days, which subsided after two weeks. The lesion underwent necrosis and sloughing, and by the end of four weeks, resolved completely (Figure 3D-3F).

\section{Case 3}

A 30 year old female patient reported with a longstanding outgrowth on the upper lip, approximately $1 \mathrm{~cm} \times 1 \mathrm{~cm}$ in size, which bled repeatedly on minor trauma. History and clinical evaluation confirmed the diagnosis of a low flow vascular malformation. The lesion was isolated with sutures and injected with $1 \mathrm{cc}$ of Polydocanol (Figure 6A and 6B). One week following sclerotherpy, there was necrosis and sloughing in the region of the lesion and edema and swelling in the surrounding areas (Figure 6C and 6D). Thereafter, there took place reduction in the swelling, with just a healing crust remaining in the region of the lesion, by the end of two weeks (Figure $6 \mathrm{E}$ and $6 \mathrm{~F})$. Complete resolution and disappearance of the VM by the end of
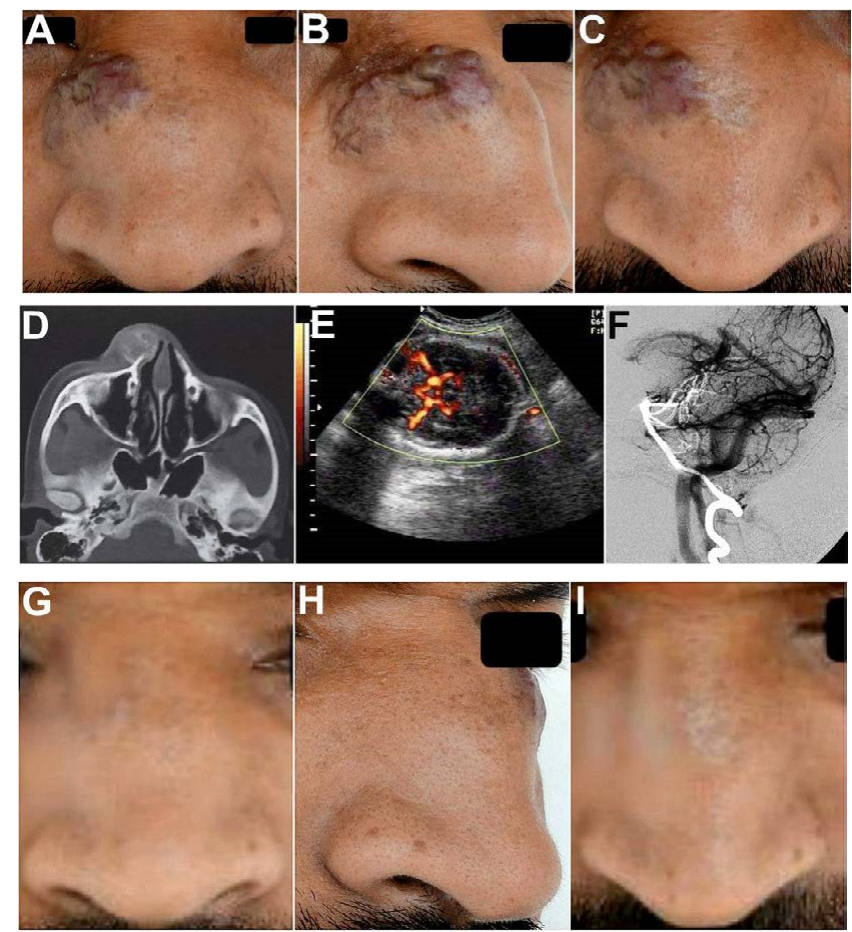

Figure 5: (A-C) Vascular Malformation (VM) of the nose in a 45 year old patient. D Non Contrast Computed Tomograph (Axial section) revealing soft tissue swelling adjacent to the nose on the right side. (E) Color Doppler Ultrasound image of the region demonstrating a mildly increased density of blood vessels within the lesion, suggestive of a Slow-flow VM. (F) Contrast enhanced Magnetic Resonance Angiography showing a vascular lesion involving branches of the Facial artery supplying lateral aspect of the nose. (G-I) 4 weeks following Single session Sclerotherapy with $1 \mathrm{cc}(30 \mathrm{mg})$ Polydocanol, showing a complete resolution and elimination of the lesion. 
the third week (Figure 6G and 6H). Colour Doppler US imaging was carried out of the Upper lip before, one month after and two months after Polydocanol sclerotherapy, which demonstrated successful regression in the vascularity of the VM and its elimination following treatment (Figure 6I and 6J). Magnetic Resonance Angiography before and two months after treatment, confirmed a successful resolution of the VM (Figure 6K and 6L).

\section{Discussion and Results}

Vascular lesions and anomalies of the maxillofacial/head and neck region were classified by Mulliken and Glowacki in 1982 [1,21], into two broad categories, Haemangiomas and Vascular malformations, based on their clinical behavior and endothelial cell characteristics. This comprehensive classification of vascular anomalies was accepted and adopted by the International Society for the Study of Vascular Anomalies (ISSVA) in 1996 (Enjolras and Mulliken 1997). In 2013, the ISSVA Classification was reinforced with an additional review on syndrome-based classification [21].

Vascular malformations (VMs) are congenital lesions resulting from developmental anomalies due to errors in vascular and lymphatic morphogenesis and are composed of stable, mature vascular endothelium abnormal morphogenesis. 31\% of VMs are found in the head and neck region [22]. These vascular lesions are present at birth, though they often go unnoticed, and grow commensurately with the child with a normal rate of endothelial cell turnover. They do not exhibit any proliferating or involution phase. They increase in size during infancy through adolescence proportionally to the overall growth of the patient. Growth may also be triggered by trauma, infection, and pubertal or other endocrine changes. Histologically there is no proliferative or involution phase and endothelial/mast cell activity is normal. Vascular channels are lined by mature endothelial cells which are surrounded by a normal reticulum. The basement membrane is a sing le cell layer

They present as a bluish/purple mass, which is soft, compressible,

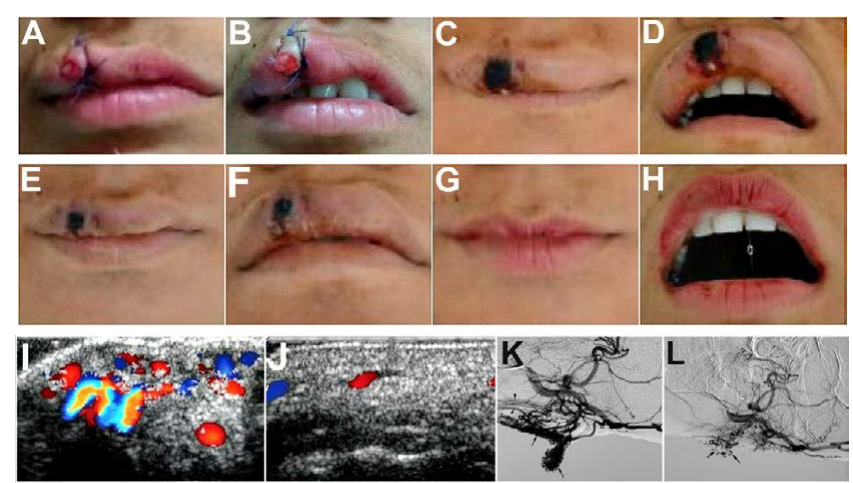

Figure 6: $(A$ and $B$ ) Isolation of the VM of the upper lip followed by injection of $1 \mathrm{cc}(30 \mathrm{mg})$ of undiluted Polydocanol in the center of the lesion. (C and D) One week following sclerotherpy, showing necrosis and sloughing in the region of the lesion and edema and swelling in the surrounding areas. ( $E$ and $F$ ) Reduction in the swelling, with just a healing crust remaining in the region of the lesion, at the end of two weeks. ( $\mathrm{G}$ and $\mathrm{H}$ ) Complete resolution and disappearance of the VM by the end of the third week. (I and J) Colour Doppler Ultrasound image of the Upper lip before and 2 months after Polydocanol sclerotherapy, demonstrating successful regression in the vascularity of the VM and its elimination following treatment. ( $\mathrm{K}$ and $\mathrm{L}$ ) Magnetic Resonance Angiography before treatment showing a vascular lesion of the upper lip (indicated by the small black arrows), and its successful resolution evident by repeat MR Angiography carried out 2 months after treatment. engorges with dependence, or increased venous pressure/Valsalva maneuver. They are usually present on the face, limbs or trunk. However, VM can also involve oro-nasopharynx, genitalia, bladder, brain, spinal cord, liver, spleen, lungs, skeletal muscles and bones.

Treatment modalities of Venous malformations include Disprin therapy, Excision or Sclerotherapy for smaller or residual/recurrent lesions [23-25] Cryotherapy for small intraoral or cutaneous lesions, Nd:YAG laser for superficial lesion or superficial component of a compound lesion, combination of Sclerotherapy and excision for larger lesions, or excision following therapeutic arterial embolization for central lesions.

As approved by the US Food and Drug Administration for uncomplicated spider veins and reticular veins in the lower extremity, an example of steady evolution in the field of phlebology is the sclerosant Polydocanol, a mixture of ethers, macrogols, and fatty alcohols that induces endothelial damage through multiple mechanisms. Repeat sessions may be necessary. Its clinical efficacy is equivalent to Sodium Tetradecyl Sulfate (STS) but with less severe adverse effects [26]. A pivotal study [27] demonstrated higher treatment success rates and statistical superiority in patient satisfaction with Polydocanol versus STS and isotonic saline. The incidence of side effects was generally lower in patients treated with Polydocanol than in those treated with STS. An experimental study has shown that Polydocanol has a much lower probability of developing tissue necrosis than any other sclerosant [28]. Furthermore, due to its anesthetic effect, it does not cause pain [29].

Sclerotherapy with Polydocanol has been carried out in rapidly growing cavernous hemangiomas mainly localised to the face $[30,31]$. 1 to 3 injections were sufficient to obtain sclerosis effect, aesthetically long term results were convincing. Severe complications were not observed [32].

Though, complications are not uncommon, the procedure is reasonably safe and there are no life threatening adverse effects seen with Polydocanol Sclerotherapy [33].

Sclerotherapy is a relatively simple, conservative, effective and inexpensive therapy which is emerging as a valuable and promising treatment modality for Vascular anomalies such as Vascular malformations and Haemangiomas. It contributes by reducing the size and vascularity of the lesions, making them more amenable to surgical excision. Multiple session sclerotherapy, usually performed at intervals of 2 to 4 weeks, followed by surgical excision is the protocol that is generally employed [34].

\section{Conclusion}

In this Case Series, a Single session Sclerotherapy by permucosal/ percutaneous intralesional endovascular instillation of 1 to $3 \mathrm{ml}$ of undiluted sclerosant liquid Polydocanol was employed, which resulted in an expeditious and complete elimination and total resolution of medium sized venous malformations involving the maxillofacial region, without the need for any surgical excision, and with minimal procedural complications, thus demonstrating the efficacy and potency of this technique. Further, single session Polydocanol injections may be preferred over multiple sitting administrations, as they are more convenient and efficacious, needing no hospitalization or repeated visits or multiple needle pricks. In view of the gratifying results achieved, it is suggested that this procedure may be considered as the first line of treatment of these challenging lesions and that larger 
Citation: Jeyaraj P (2017) Efficacy of Single Session Sclerotherapy with Undiluted Polydocanol, in Complete Elimination of Cutaneous as Well as Mucosal Venous Malformations of the Maxillofacial Region. J Cytol Histol 8: 464. doi: 10.4172/2157-7099.1000464

Page 6 of 6

studies may be undertaken to corroborate these findings and employ it as an established protocol for such lesions.

\section{Compliance with Ethical Standards}

\section{Disclosure of potential conflicts of interest}

The author of this article has not received any research grant, remuneration, or speaker honorarium from any company or committee whatsoever, and neither owns any stock in any company. The author declares that she does not have any conflict of interest.

\section{Research involving human participants and/or animals}

All procedures performed on the patients (human participants) involved were in accordance with the ethical standards of the institution and/or national research committee, as well as with the 1964 Helsinki declaration and its later amendments and comparable ethical standards.

\section{Ethical approval}

Although the use of intralesional injection of Polydocanol is a well-established and widely used treatment procedure for vascular malformations, the novel technique of its use as a single session sclerotherapy protocol, is described for the first time. Hence, this study was evaluated and approved by an Institutional ethical committee.

\section{Informed Consent}

Informed consent was obtained from all the individual participants in this study.

\section{Funding}

This study was not funded by any organization/society.

\section{References}

1. Mulliken JB, Glowacki J (1982) Hemangiomas and vascular malformations in infants and children: A classification based on endothelial characteristics. Plast Reconstr Surg 69: 41-44.

2. Jackson IT, Carreño R, Potparic Z, Hussain K (1993) Hemangiomas, vascular malformations, and lymphovenous malformations: Classification and methods of treatment. Plast Reconstr Surg 91: 121-130.

3. Lee CH, Chen SG (2005) Direct percutaneous ethanol instillation for treatment of venous malformation in the face and neck. Br J Plast Surg 58: 107-108.

4. Lee KB, Kim DI, Oh SK, Do YS, Kim KH, et al. (2008) Incidence of soft tissue injury and neuropathy after embolo/sclerotherapy for congenital vascular malformation. J Vasc Surg 48: 1286-1291.

5. Apfelberg DB, Maser MR, Lash H, White DN (1985) Benefits of the carbon dioxide laser in oral hemangioma excision. Plast Reconstr Surg 75: 46-50.

6. Landthaler M, Hohenleutner U, el-Raheem TA (1995) Laser therapy of childhood haemangiomas. Br J Dermatol (1995) 133: 275-281.

7. Ezekowitz RA, Mulliken JB, Folkman J (1992) Interferon alfa-2a therapy for lifethreatening hemangiomas of infancy. N Engl J Med 326: 1456-1463.

8. Braun IF, Levy S, Hoffman JC Jr (1985) The use of transarterial microembolization in the management of hamangiomas of perioral region. $J$ Oral Maxillofac Surg 43: 39-48.

9. Jasim ZF, Woo WK, Handley JM (2004) Long-pulsed (6-ms) pulsed dye laser treatment of rosacea-associated telangiectasia using subpurpuric clinical threshold. Dermatol Surg 30: 37-40.

10. Itinteang $T$, Withers $A H$, Leadbitter $P$ (2011) Pharmacologic therapies for infantile hemangioma: is there a rational basis? Plast Reconstr Surg 128: 499-507.

11. Spiteri Cornish K, Reddy AR (2011) The use of propranolol in the management of periocular capillary hemangioma-a systematic review. Eye (Lond) 25: 12771283.
12. Tisi PV, Beverley C, Rees A (2006) Injection Sclerotherapy for varicose veins. Cochrane database Syst Rev 18: CD001732.

13. Spence J, Krings T, terBrugge KG, da Costa LB, Agid R (2010) Percutaneous sclerotherapy for facial venous malformations: Subjective clinical and objective MR imaging followup results. Am J Neuroradiol 31: 955-960.

14. Buckmiller LM, Richter GT, Suen JY (2010) Diagnosis and management of hemangiomas and vascular malformations of the head and neck. Oral Dis 16: 405-418.

15. Jackson IT, Keskin M, Yavuzer R, Kelly CP (2005) Compartmentalization of massive vascular malformations. Plast Reconstr Surg 115: 1021-1023.

16. Chen WL, Yang ZH, Bai ZB, Wang YY, Huang ZQ, et al. (2008) A pilot study on combination compartmentalisation and sclerotherapy for the treatment of massive venous malformations of the face and neck. J Plast Reconstr Aesthet Surg 61: 148-153.

17. Sachin K, Rashmi S, Manish S, Siddhartha W, Uday L (2013) Haemangiomas and venous malformations of the head and neck: A retrospective analysis of endovascular management in 358 patients. Indian J Plast Surg 46: 109-116.

18. Woods JE (1987) Extended use of sodium tetradecyl sulfate in treatment of hemangiomas and other related conditions. Plast Reconstr Surg 79: 542-549.

19. Burrows PE (2013) Endovascular treatment of slow-flow vascular malformations. Tech Vasc Interv Radiol 16: 12-21.

20. Blaise S, Charavin-Cocuzza M, Riom H, Brix M, Seinturier C, et al. (2011) Treatment of low-flow vascular malformations by ultrasound-guided sclerotherapy with polidocanol foam: 24 cases and literature review. Eur J Vasc Endovasc Surg 41: 412-417.

21. Ethunandan, M, Pratt CA, Morrison, A (2006) Multiple Synchronous and Metachronous Neoplasms of the Parotid Gland: The Chichester Experience. British Journal of Oral and Maxillofacial Surgery 44, 397-401.

22. Neil S. Sadick, Nils Krueger (2012) Vascular Lesions. Cosmetic Dermatology 25: 108-109.

23. O'Donovan JC, Donaldson JS, Morello FP, Pensler JM, Vogelzang RL, et al. (1997) Symptomatic hemangiomas and venous malformations in infants, children, and young adults: Treatment with percutaneous injection of sodium tetradecyl sulfate. AJR Am J Roentgenol 169: 7239-7240.

24. Lee CH, Chen SG (2005) Direct percutaneous ethanol instillation for treatment of venous malformation in the face and neck. Br J Plast Surg 58: 10738-10740.

25. Berenguer B, Burrows PE, Aurakowski D, Mulliken JB (1999) Sclerotherapy of Craniofacial venous malformation: Complications and results. Plast Reconstr Surg 104: 1-15.

26. Conrad P, Malouf GM, Stacey MC (1995) The Australian polidocano (aethoxysklerol) study. results at 2 years. Dermatol Surg 21: 334-338.

27. Rabe E, Schliephake D, Otto J (2010) Sclerotherapy of telangiectases and reticular veins: a double-blind, randomized, comparative clinical trial of polidocanol, sodium tetradecyl sulphate and isotonic saline (EASI study). Phlebology 25: 124-131.

28. Kobayashi S, Crooks S, Eckmann DM (2006) Dose- and time-dependent liquid sclerosant effects on endothelial cell death. Dermatol Surg 32: 1444-1452.

29. Guex JJ, Schliephake DE, Otto J (2010) The French polidocanol study on longterm side effects: a survey covering 3,357 patient years. Dermatol Surg 36: 993-903.

30. Winter H, Drager E, Steny W (2000) Sclerotherapy for treatment of hemangiomas In: Dermatol surg 26: 105-108.

31. Carvalho RA, Neto V (2010) Polidocanol sclerotherapy in treatment of pyogenic granuloma. In: Dermatol Surg 36: 1068-1070.

32. Jin Y, Lin X, Chen LW, Hu X, Ma G, et al. (2009) Craniofacial venous malformations: magnetic resonance imaging features that predict treatment outcome. J Oral Maxillofac Surg 67: 2388-2396.

33. Berenguer B, Burrows PE, Zurakowski D, Mulliken JB (1999) Sclerotherapy of craniofacial venous malformations: Complications and results. Plast Reconstr Surg 104: 111

34. Sachin K, Rashmi S, Manish S, Siddhartha W (2013) Haemangiomas and venous malformations of the head and neck: A retrospective analysis of endovascular management in 358 patients. Indian Journal of Plastic Surgery 46: 109-116. 